\title{
Orthodontic Inventory for Management of Nickel-sensitive Patients: An In Vitro Study
}

\author{
Mukesh Kumar ${ }^{1}$, Sommya Kumari ${ }^{2}$, Konark $^{3}$, Anju Singh ${ }^{4}$, Pankaj Kumar $^{5}$, Pragyan Das ${ }^{6}$
}

\begin{abstract}
Aim: To compare the frictional resistance produced by titanium and stainless steel (SS) brackets with various orthodontic wires used in sliding mechanics. To evaluate surface texture of brackets and wires using scanning electron microscopy (SEM).

Materials and methods: A total of 40 canine brackets of titanium and SS having $0.022^{\prime \prime}$ slot were tested for static and kinetic friction, with SS, low-friction titanium-molybdenum alloy (TMA), timolium and titanium-niobium (Ti-Nb) wires $\left(0.019^{\prime \prime} \times 0.025^{\prime \prime}\right)$, using a universal testing machine. To evaluate the surface roughness, the brackets and wires were subjected to SEM before and after testing.

Results: Titanium brackets showed greater mean static and kinetic frictional forces compared to SS brackets with all the wires that were tested. It was lowest with timolium wire followed by SS wire, low-friction TMA and highest with Ti-Nb wire. The SEM indicated greater surface roughness of titanium bracket slot compared to the SS bracket slot, and titanium wires showed greater surface roughness compared to the SS wire.

Conclusion: The SS bracket with SS wire would produce efficient results during retraction in sliding mechanics. However, in nickel-sensitive patients where titanium brackets are used as an alternative, the wire of choice would be timolium wire. Low-friction TMA and Ti-Nb wires would be the secondary wires of choice.

Clinical significance: Nickel sensitivity and frictional resistance remain a matter of concern during orthodontic treatment for the patients and orthodontist, respectively. This study highlights the orthodontic wire of choice to reduce frictional resistance while using titanium brackets in nickel-sensitive patients.
\end{abstract}

Keywords: Friction, Nickel sensitivity, Titanium brackets.

The Journal of Contemporary Dental Practice (2020): 10.5005/jp-journals-10024-2826

\section{INTRODUCTION}

Orthodontic tooth movements are achieved when the brackets slide in relation to the arch wire for correction of malocclusion. Frictional resistance is encountered wherever sliding occurs. ${ }^{1}$ This frictional resistance must be controlled in order to apply optimal forces. $^{2}$ There are two types of friction: static and kinetic. Static friction opposes any applied force. Kinetic friction, which usually is less than static friction, opposes the direction of motion of the object. Currently, the "gold standard" consists of an arch wirebracket couple composed solely of SS that displays the least friction. However, SS orthodontic appliances are no longer free of potential problems. ${ }^{3}$ Various studies have found liberation of nickel from SS alloys. ${ }^{2-7}$ Genelhu et al. ${ }^{8}$ found that an oral inflammatory response is induced by corrosion of orthodontic appliances and subsequent nickel release. This hypersensitivity reaction is manifested as nickel-induced allergic contact stomatitis in some patients. Ortiz et al. found the greatest toxicity to cultured human fibroblasts and cellular DNA by SS alloys. ${ }^{4}$ Park and Shearer reported an average release of $40 \mu \mathrm{g}$ of nickel per day from a simulated full-mouth fixed appliance. ${ }^{9}$ Sfondrini et al. in a study stated nickel to be a strong immunologic sensitizer and a cause of allergic contact stomatitis in some orthodontic patients. ${ }^{10}$ Gursoy et al. pointed out the role of nickel accumulation and epithelial cell proliferation in orthodontic treatment-induced gingival overgrowth. ${ }^{11}$ Furthermore, SS brackets also undergo corrosion and ferromagnetism, thus, distorting magnetic resonance imaging and computed tomography scan images. ${ }^{12,13}$ For this reason, various choices of brackets are available for nickel-sensitive patients like polycarbonate, ceramic, and titanium brackets. Polycarbonate brackets easily undergo deformation, absorb water, and are dimensionally unstable. Ceramic

\footnotetext{
${ }^{1}$ Faculty of Dentistry, Vardhman Institute of Medical Sciences, Pawapuri, Bihar, India

${ }^{2}$ Department of Prosthodontics and Crown and Bridge, Buddha Institute of Dental Sciences and Hospital, Patna, Bihar, India

${ }^{3}$ Department of Conservative Dentistry and Endodontics, Patna Dental College and Hospital, Patna, Bihar, India

${ }^{4}$ Faculty of Dentistry, Nalanda Medical College and Hospital, Patna, Bihar, India

${ }^{5}$ Department of Conservative Dentistry and Endodontics, Awadh Dental College and Hospital, Jamshedpur, Jharkhand, India

${ }^{6}$ Department Oral Medicine and Radiology, Awadh Dental College and Hospital, Jamshedpur, Jharkhand, India
}

Corresponding Author: Sommya Kumari, Department of Prosthodontics and Crown and Bridge, Buddha Institute of Dental Sciences and Hospital, Patna, Bihar, India, Phone: +91 8969859326, e-mail: sommyakumari@gmail.com

How to cite this article: Kumar M, Kumari S, Konark, et al. Orthodontic Inventory for Management of Nickel-sensitive Patients: An In Vitro Study. J Contemp Dent Pract 2020;21(6):645-650.

Source of support: Nil

Conflict of interest: None

brackets are brittle and expensive. They produce greater friction and enamel wear owing to their hardness and can also fracture. Debonding may result in damage to the enamel. ${ }^{13}$ Apart from that titanium brackets are nickel free, nonmagnetic and have excellent corrosion resistance and biocompatibility., ${ }^{42,14}$ Titanium is known to have a rough surface texture. ${ }^{14-16}$ So it is expected that the friction may be higher during sliding mechanics. This may enhance the

(c) The Author(s). 2020 Open Access This article is distributed under the terms of the Creative Commons Attribution 4.0 International License (https://creativecommons. org/licenses/by-nc/4.0/), which permits unrestricted use, distribution, and non-commercial reproduction in any medium, provided you give appropriate credit to the original author(s) and the source, provide a link to the Creative Commons license, and indicate if changes were made. The Creative Commons Public Domain Dedication waiver (http://creativecommons.org/publicdomain/zero/1.0/) applies to the data made available in this article, unless otherwise stated. 
need for greater force application and may also have adverse effects on anchorage needs. Hence, it is important to study the sliding properties of titanium brackets during retraction for achieving frictionless tooth movement without hampering anchorage during orthodontic treatment. No previous study has investigated the frictional behavior of titanium brackets with nickel-free orthodontic wires for nickel-hypersensitive patients. Therefore, the aim of this study was to compare the frictional resistance of titanium and SS brackets with various wires used in sliding mechanics for closure of extraction spaces. So that the SS brackets and wires could be avoided in nickel-sensitive patients without significantly increasing the frictional resistance.

\section{Materials and Methods}

The frictional resistance and SEM study was conducted at Praj Metallurgical Laboratory, Pune. The SS (ORMCO, USA) and titanium brackets (ORMCO, USA) used were standard twin brackets (022" $\times 028^{\prime \prime}$ ) for maxillary right canine having four tie wings for engagement of SS ligature. Titanium brackets consisted of two parts, the base (commercially pure Ti grade II) and the wing (Ti-6Al-4V alloy) joined together by laser welding. ${ }^{17}$ They were formulated to be nickel-free.4 Due to the lack of availability of titanium brackets in standard edgewise configuration, all brackets (titanium and SS) were of preadjusted edgewise configuration and had $-3^{\circ}$ torque and $10^{\circ}$ angulation.

The study sample consisted of 80 canine brackets $\left(022^{\prime \prime} \times 028^{\prime \prime}\right)$ of two different materials.

- Stainless steel $(n=40)$

- Titanium $(n=40)$

Four types of orthodontic wires were tested.

- Stainless steel (18-8 SS) (G\&H Wire Company, USA)

- Low-Friction Beta-Titanium (TMA) (ORMCO, USA)

- Timolium (Alpha-Beta-Titanium or Titanium, Aluminum and Vanadium Alloy) (T.P. Orthodontics, USA)

- The Ti-Nb Alloy

Ten brackets, each of both the bracket materials, were tested with four different types of orthodontic wire $\left(0.019^{\prime \prime} \times 0.025^{\prime \prime}\right)$. These bracket-wire combinations were divided into eight groups.

\begin{tabular}{lll}
\hline Group I & SS-SS & $(n=10)$ \\
Group II & SS-low-friction TMA & $(n=10)$ \\
Group III & SS-timolium & $(n=10)$ \\
Group IV & SS-Ti-Nb (ORMCO, USA) & $(n=10)$ \\
Group V & Titanium-SS & $(n=10)$ \\
Group VI & Titanium-low-friction TMA & $(n=10)$ \\
Group VII & Titanium-Timolium & $(n=10)$ \\
Group VIII & Titanium-Ti-Nb & $(n=10)$ \\
\hline
\end{tabular}

Therefore, a total of 80 samples comprising of 10 samples each of 08 bracket-arch wire combinations were tested in totality. A total of 80 wire segments of $4.0 \mathrm{~cm}$ length (10 wire samples each of all the four materials using both the SS and titanium brackets) were tested.

An experimental model was prepared before the commencement of testing. The brackets were bonded to an SS bar of $100 \times 15 \times 1 \mathrm{~mm}$, using cyanoacrylate adhesive. A guide for reproducing the bond position was marked in the midline of each bar which was parallel to the long axis of the bar. A $0.021 \times 0.028$ inch
SS wire was used to ensure the bracket slot be parallel to the length of steel bar as described by Doshi et al. ${ }^{18}$

Total eight groups of bracket-wire combinations were made. First four groups were having SS brackets tested with SS, TMA, timolium, and $\mathrm{Ti}-\mathrm{Nb}$ wires, respectively, and similarly groups $\mathrm{V}$ to VIII were combination of titanium brackets with SS, TMA, timolium, and $\mathrm{Ti}-\mathrm{Nb}$ wires, respectively.

To measure surface roughness, the prepared experimental models were examined both prior to and after friction testing using SEM at 1000× magnification (QUANTA 200; E SEM, Bengaluru, India, with $20 \mathrm{kV}$ ).

The frictional resistance was measured using the universal testing machine. The bracket-wire assembly was vertically mounted and clamped to the jaws of the universal testing machine (LR 50K; LLYOD instruments). The wire protruding from the bracket was carefully clamped to the movable crosshead. Care was taken not to twist the test wire. The load cell was calibrated at $10 \mathrm{~N}$. The wire was drawn through the bracket slot at a rate of $3 \mathrm{~mm}$ per minute with the up and down movement of crosshead.

The bracket-arch wire friction was measured using the universal testing machine in dry state. To measure the friction, the wire specimen was drawn through the bracket. A total of 40 canine brackets of titanium and 40 canine brackets of SS $\left(022^{\prime \prime} \times 028^{\prime \prime}\right)$ were tested with 10 arch wire each of SS, low-friction TMA, timolium, and $\mathrm{Ti}-\mathrm{Nb}$. Therefore, all the eight bracket-arch wire combinations were tested 10 times and the average was recorded. Each bracket-wire combination was tested only once to eliminate the wear influence.

A computer attached to the universal testing machine recorded the resulting frictional force automatically for all bracket and wire combinations. For every traction test over a distance of 10 $\mathrm{mm}$ at a speed of $3 \mathrm{~mm} /$ minute frictional forces recorded were the maximum force needed to move the bracket along the wire (static friction) and the mean frictional force registered at $10 \mathrm{~mm}$ of movement (kinetic friction).

\section{Results}

The present study compared the frictional resistance of two different bracket materials with four different wires. A total of eight bracket-wire combinations were tested 10 times each.

The results for mean static frictional forces are given in Table 1. For titanium brackets, the mean static frictional force values ( $p<$ $0.001)$ were lowest with timolium wire $(0.84 \pm 0.21 \mathrm{~N})$ followed by SS wire $(1.03 \pm 0.15 \mathrm{~N})$ then low friction TMA and highest with $\mathrm{Ti}-\mathrm{Nb}$ wire $(1.13+0.09 \mathrm{~N})$. Group VII $<$ group V $<$ group VI $<$ group VIII, for SS brackets, the mean static frictional force values $(p<0.001)$ were

Table 1: Comparison of static friction (Newton) in eight groups

\begin{tabular}{llll}
\hline & \multicolumn{3}{c}{ Static friction } \\
\cline { 2 - 4 } Groups & Min-Max & Mean \pm SD & $95 \% \mathrm{CI}$ \\
\hline Group I & $0.38-0.59$ & $0.53 \pm 0.063$ & $0.49-0.58$ \\
Group II & $0.51-1.2$ & $0.94 \pm 0.23$ & $0.78-1.11$ \\
Group III & $0.42-0.73$ & $0.56 \pm 0.11$ & $0.48-0.63$ \\
Group IV & $0.8-1.2$ & $1.02 \pm 0.13$ & $0.92-1.11$ \\
Group V & $0.79-1.2$ & $1.03 \pm 0.15$ & $0.92-1.13$ \\
Group VI & $0.72-1.3$ & $1.12 \pm 0.18$ & $0.99-1.25$ \\
Group VII & $0.6-1.2$ & $0.84 \pm 0.21$ & $0.69-0.99$ \\
Group VIII & $1-1.3$ & $1.13 \pm 0.09$ & $1.06-1.2$ \\
\hline
\end{tabular}

$F=23.046 ;{ }^{*} p<0.001$ 
lowest with SS wire $(0.53 \pm 0.063 \mathrm{~N})$ followed by timolium wire $(0.56$ $\pm 0.11 \mathrm{~N})$ then low-friction TMA $(0.94 \pm 0.23 \mathrm{~N})$ and highest with Ti-Nb wire $(1.02 \pm 0.13 \mathrm{~N})$. Group I < group III < group II < group IV, on comparison with SS brackets, titanium brackets showed higher mean static frictional force values with SS wire and timolium wire, and the difference was statistically significant (Table 2). Also the mean frictional forces were higher with low friction $\mathrm{TMA}$ and $\mathrm{Ti}-\mathrm{Nb}$ wires, but this difference was not significant. The results for mean kinetic frictional forces are given in Table 3. For titanium brackets, the mean kinetic frictional force values $(p<0.001)$ were lowest with timolium wire $(0.50 \pm 0.14 \mathrm{~N})$ followed by SS wire $(0.52 \pm 0.10 \mathrm{~N})$, then low-friction TMA $(0.52 \pm 0.11 \mathrm{~N})$ and highest with $\mathrm{Ti}-\mathrm{Nb}$ wire $(0.58 \pm 0.07 \mathrm{~N})$. Group VII $<$ group V $<$ group VI $<$ group VIII, for SS brackets the mean kinetic frictional force values $(p<0.001)$ were lowest with SS wire $(0.17 \pm 0.04 \mathrm{~N})$ then timolium $(0.31 \pm 0.03 \mathrm{~N})$ followed by low-friction TMA $(0.43 \pm 0.14 \mathrm{~N})$ and highest with $\mathrm{Ti}-\mathrm{Nb}$ wire $(0.49 \pm 0.09 \mathrm{~N})$ (Table 4$)$. Group I < group III < group II < group IV, titanium brackets showed higher mean kinetic frictional force values compared to SS brackets in all the groups. But the frictional resistance between titanium brackets and timolium wires was not statistically significantly high as that between SS brackets and SS

Table 2: Post hoc tests (pair wise comparison of static friction)

\begin{tabular}{lccc}
\hline Pair & Mean difference & p value & $95 \% \mathrm{CI}$ \\
\hline Groups I-II & -0.41 & $<0.001^{*}$ & $-0.624-0.192$ \\
Groups I-III & -0.021 & 1.000 & $-0.237-0.195$ \\
Groups I-IV & -0.48 & $<0.001^{*}$ & $-0.694-0.263$ \\
Groups I-V & -0.49 & $<0.001^{*}$ & $-0.704-0.273$ \\
Groups II-III & 0.39 & $<0.001^{*}$ & $0.166-0.608$ \\
Groups II-IV & -0.07 & 0.973 & $-0.292-0.15$ \\
Groups II-VI & -0.18 & 0.218 & $-0.397-0.045$ \\
Groups III-IV & -0.46 & $<0.001^{*}$ & $-0.679-0.237$ \\
Groups III-VII & -0.28 & $0.004^{*}$ & $-0.503-0.061$ \\
Groups IV-VIII & -0.12 & 0.734 & $-0.336-0.106$ \\
Groups V-VI & -0.09 & 0.879 & $-0.316-0.126$ \\
Groups V-VII & 0.19 & 0.163 & $-0.035-0.407$ \\
Groups V-VIII & -0.11 & 0.814 & $-0.326-0.116$ \\
Groups VI-VII & 0.28 & $0.004^{*}$ & $0.06-0.502$ \\
Groups VI-VIII & -0.01 & 1.000 & $-0.231-0.211$ \\
Groups VII-VIII & -0.29 & $0.002^{*}$ & $-0.512-0.07$ \\
\hline
\end{tabular}

*Strongly significant

Table 3: Comparison of kinetic friction (Newton) in eight groups

\begin{tabular}{llll}
\hline & \multicolumn{3}{c}{ Kinetic friction } \\
\cline { 2 - 4 } Groups & Min-Max & Mean $\pm S D$ & $95 \% \mathrm{CI}$ \\
\hline Group I & $0.10-0.2$ & $0.17 \pm 0.04$ & $0.14-0.19$ \\
Group II & $0.27-0.63$ & $0.43 \pm 0.14$ & $0.33-0.53$ \\
Group III & $0.25-0.35$ & $0.31 \pm 0.03$ & $0.29-0.33$ \\
Group IV & $0.35-0.6$ & $0.49 \pm 0.09$ & $0.42-0.55$ \\
Group V & $0.35-0.65$ & $0.52 \pm 0.10$ & $0.45-0.59$ \\
Group VI & $0.35-0.63$ & $0.52 \pm 0.11$ & $0.44-0.59$ \\
Group VII & $0.31-0.65$ & $0.50 \pm 0.14$ & $0.40-0.6$ \\
Group VIII & $0.50-0.7$ & $0.58 \pm 0.07$ & $0.53-0.62$ \\
\hline F=
\end{tabular}

$F=19.591 ;{ }^{*} p<0.001$ wires. The SEM examination indicated greater surface roughness of titanium bracket slot compared to SS bracket slot and greater surface roughness of timolium, low-friction TMA, and Ti-Nb wires compared to SS wire both before and after testing (Figs 1 to 6 ).

\section{Discussion}

Orthodontic appliances remain in the mouth for long in a potentially corrosive environment and release metallic ions into the tissues and fluids of patients wearing them. ${ }^{4}$ Currently, the gold standard consists of an arch wire-bracket couple composed solely of SS that displays the least friction. ${ }^{3}$ Clinical manifestations such as glossitis, metal taste, gingivitis, peeling lips, erythema multiforme, and gingival hypertrophy damage to DNA have been observed. Such manifestations are usually associated with the inflammatory response induced by the release of heavy metals, and major emphasis has been placed on nickel. ${ }^{4,8,11}$ Also the union between nickel atoms and the intermetallic compounds is not strong, leading to nickel release from the alloy's surface causing nickel allergy. Inflammatory response to nickel is considered as type IV hypersensitivity and is manifested as nickel allergic contact stomatitis. ${ }^{11}$ For this reason alloys, other than SS, with greater biocompatibility, more stability in the oral environment, and less susceptibility to corrosion have been introduced.

\section{Polycarbonate, Ceramic, Titanium Brackets}

The SS and ceramic brackets are strong and dimensionally stable during treatment. The plastic materials show evidence of permanent deformation during treatment owing to their relatively lower strength. The force produced by arch wire and occlusal loading propagates the stress, causing failure. ${ }^{4}$

The torsional force produced by arch wires causes distortion of polycarbonate brackets. An additional property of inclination toward water absorption causes discoloration and staining which is undesirable. The staining property and distortion of these brackets pose a limitation to its clinical use. ${ }^{4}$

A high occurrence ceramic bracket fracture causes distress. The brittle nature of ceramic bracket makes it prone to fracture and even

Table 4: Pair wise comparison of kinetic friction (post hoc test)

\begin{tabular}{lccl}
\hline Pair & Mean difference & $p$ value & $95 \% \mathrm{CI}$ \\
\hline Groups I-II & -0.27 & $<0.001^{* *}$ & $-0.4-0.13$ \\
Groups I-III & -0.14 & $0.035^{*}$ & $-0.276-0.006$ \\
Groups I-IV & -0.32 & $<0.001^{* *}$ & $-0.454-0.184$ \\
Groups I-V & -0.36 & $<0.001^{* *}$ & $-0.491-0.221$ \\
Groups II-III & 0.12 & $0.097^{+}$ & $-0.011-0.259$ \\
Groups II-IV & -0.05 & 0.916 & $-0.189-0.081$ \\
Groups II-VI & -0.08 & 0.532 & $-0.219-0.051$ \\
Groups III-IV & -0.18 & $0.003^{*}$ & $-0.313-0.043$ \\
Groups III-VII & -0.19 & $0.001^{* *}$ & $-0.329-0.059$ \\
Groups IV-VIII & -0.09 & 0.441 & $-0.225-0.045$ \\
Groups V-VI & 0.01 & 1.000 & $-0.128-0.142$ \\
Groups V-VII & 0.02 & 1.00 & $-0.114-0.156$ \\
Groups V-VIII & -0.05 & 0.923 & $-0.188-0.082$ \\
Groups VI-VII & 0.01 & 1.000 & $-0.121-0.149$ \\
Groups VI-VIII & -0.06 & 0.862 & $-0.195-0.075$ \\
Groups VII-VIII & -0.07 & 0.684 & $-0.209-0.061$ \\
\hline
\end{tabular}

${ }^{+}$Suggestive significance, ${ }^{*}$ Moderately significant, ${ }^{* *}$ Strongly significant 


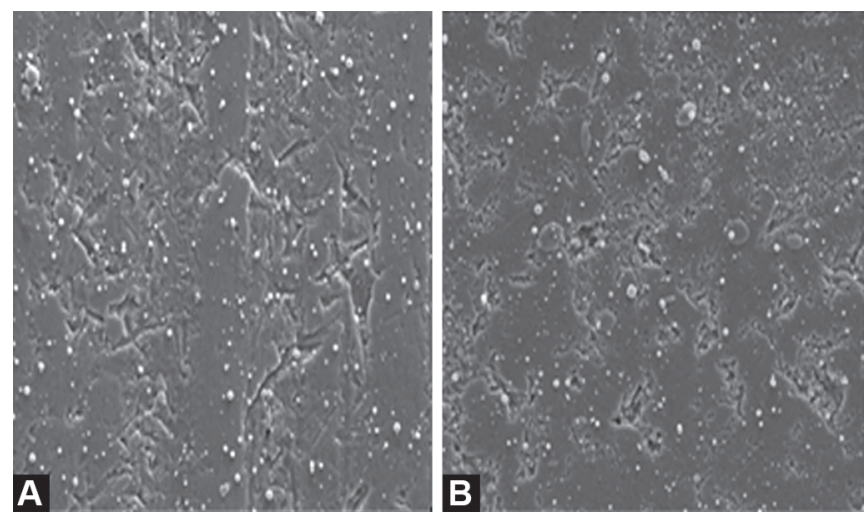

Figs $1 \mathrm{~A}$ and B: Titanium bracket slot showing a rough granular surface with depressions both before and after testing. [Pretesting $(A)$ and posttesting (B) SEM image of titanium bracket slot at 1000×]

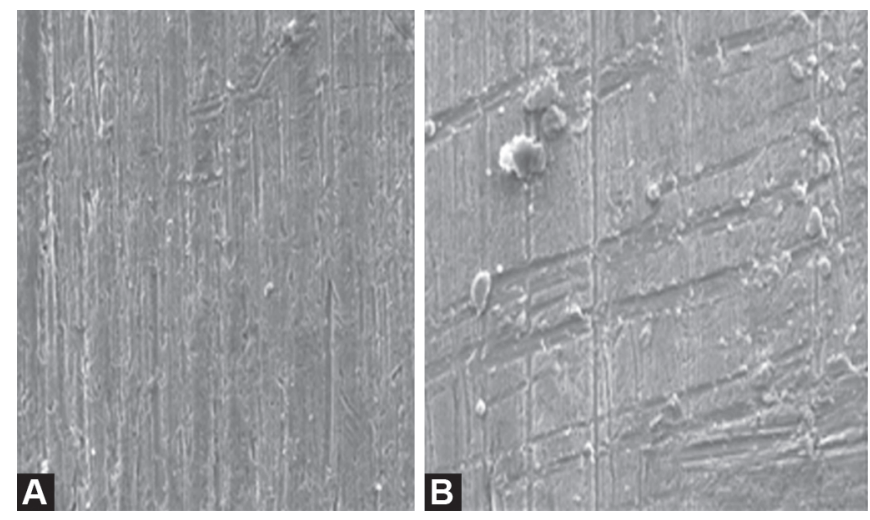

Figs $3 \mathrm{~A}$ and $\mathrm{B}$ : Titanium niobium wire showing a striated pattern before testing on which deep vertical and horizontal grooves were seen after testing. [Pretesting (A) and posttesting (B) SEM image of Ti-Nb wire at $1000 \times]$

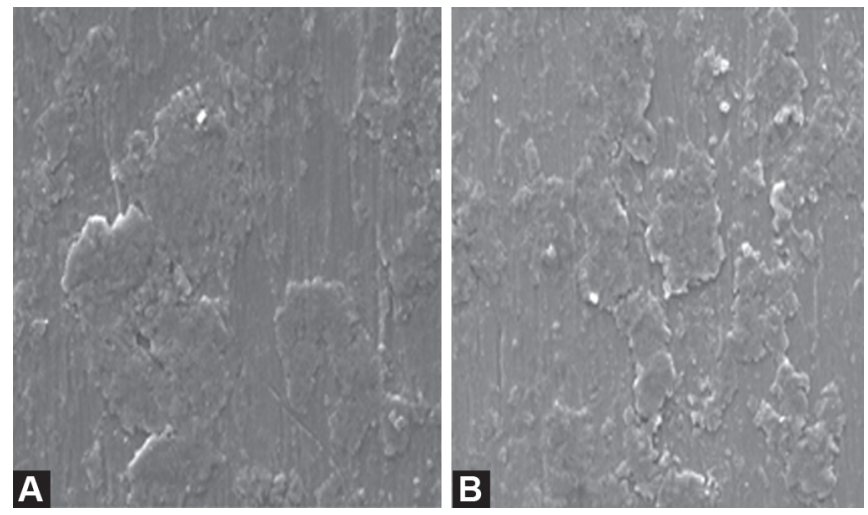

Figs $5 \mathrm{~A}$ and $\mathrm{B}$ : Timolium wire showing irregular patchy appearance before testing and no significant difference in appearance posttesting. [Pretesting (A) and posttesting (B) SEM image of timolium wire at 1000X]

a minute surface flaw dramatically reduces the fracture load. These fractured brackets have reduced control over tooth movement and therefore deliver an ineffective tooth movement. This condition increases the overall treatment time. There exists a fear of inhaling or ingesting the fractured pieces of bracket.

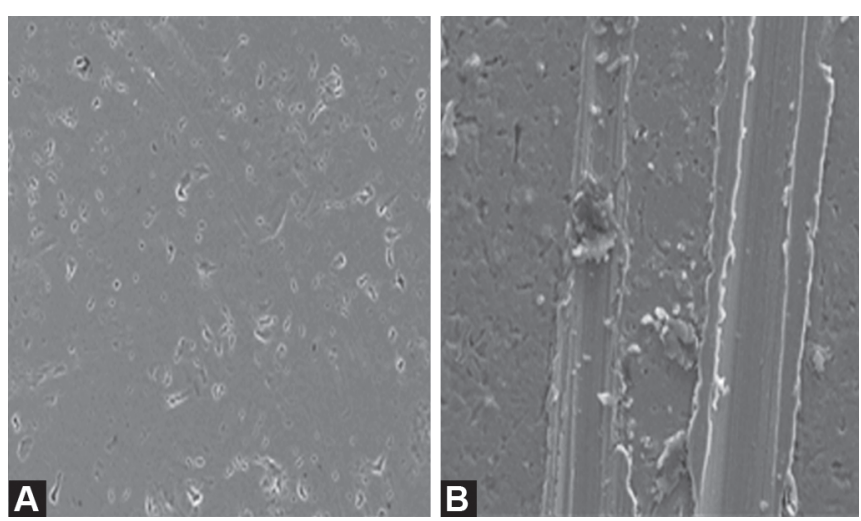

Figs $2 \mathrm{~A}$ and B: Stainless steel bracket slot showing smooth surface before testing and few striations after testing. [Pretesting (A) and posttesting (B) SEM image of SS bracket slot at 1000× ]

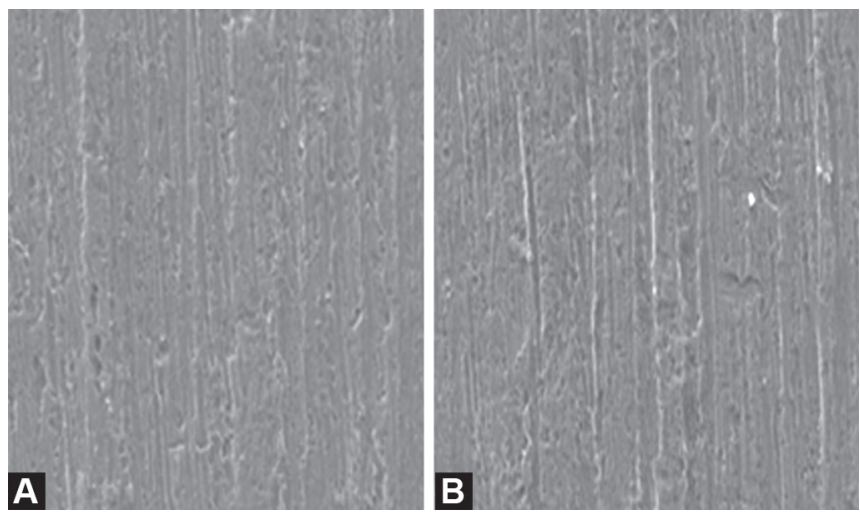

Figs 4A and B: Low-friction TMA wire showing an uneven pitted and striated surface texture both pre- and posttesting. [Pretesting $(A)$ and posttesting (B) SEM image of TMA wire at 1000× ]

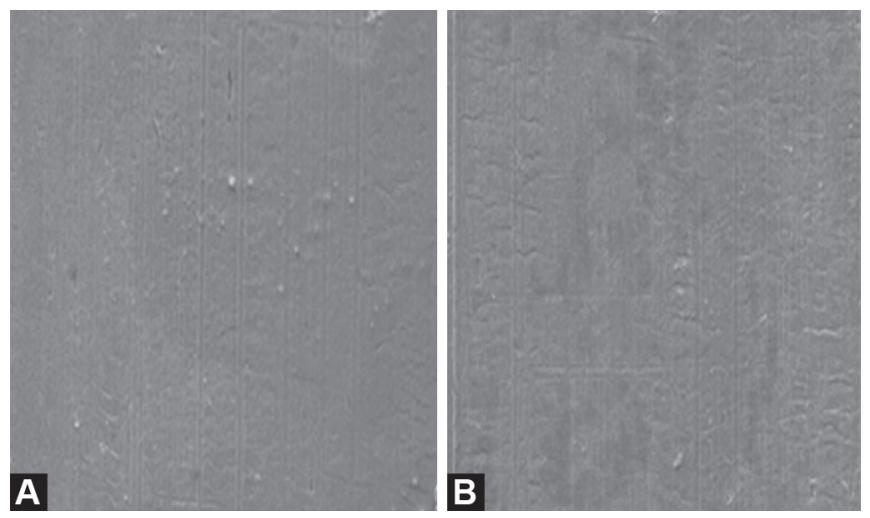

Figs $6 A$ and $B$ : Stainless steel wire showing smooth surface morphology before and mild streaks on its surface after testing. [Pretesting $(A)$ and posttesting (B) SEM image of SS wire at 1000X]

The bonding of ceramic bracket is aided by surface coating of silane coupling agent at the bracket base. This surface coating exponentially increases the bond strength, making the enamel surface susceptible to stress and/or fracture during bracket debonding. The endodontically treated tooth and tooth with 
large restorations are comparatively more prone to fracture under such stress. An additional drawback of ceramic bracket includes abrasion of opposing tooth due to its hardness. ${ }^{4}$ Excessively high bond strength of ceramic brackets compromises the enamel safety margin causing enamel fracture.

In this study, we tested various combinations of materials to find better alternative toward allergic reactions without causing any significant change in mechanics and minimal frictional resistance. The present effort evaluates commercially pure Ti brackets and Ti-based wires for such an alternative. Beta-titanium is described as having an excellent balance of properties, including high spring back, low stiffness, high formability, and the ability of direct welding and being nickel free. However, a drawback is its high coefficient of friction. ${ }^{19}$ The SS and commercially pure (cp) titanium brackets both tested were covered with a protective surface oxide layer. The chemical constituents of this passive layer on SS bracket consisted of chromium oxide, and for titanium bracket the passive layer is mostly (rutile) titanium dioxide. ${ }^{3,14}$ Kusy et al. found in a study that this passive layer did not breakdown and withstood the stress, although resistance to sliding increased with increase in angulation and normal force. Hence, the passive oxide layers on SS and titanium brackets provide a good medium for sliding mechanics. ${ }^{12}$

Doshi et al. in their study found a strong correlation between bracket slot roughness and frictional resistance. ${ }^{18}$ Friction is greater between two surfaces of the same material than two surfaces of different materials, and frictional forces are largely due to the atomic and molecular forces of attraction at the small contact areas between materials. ${ }^{12}$ Thus, titanium brackets showed high friction with $\mathrm{Ti}-\mathrm{Nb}$ wire and low-friction TMA wire. High-frictional values could be explained on the basis of SEM examination of beta-titanium wires that revealed rough surface texture. The $\mathrm{Ti}-\mathrm{Nb}$ wire had a striated surface pattern on which deep horizontal and vertical scratches were seen after testing (Fig. 5), whereas lowfriction TMA had an uneven pitted and striated surface texture due to ion implantation before and after testing (Fig. 6). These features could be attributed to adherence or cold welding of titanium to the dies or rollers during wire processing. This surface roughness contributes to the high friction during sliding. ${ }^{19,20}$ In this study, it was seen that both titanium and SS brackets produced greater friction with low-friction TMA than with SS wire. Similar result was obtained by Michelberger et al. ${ }^{21}$ who tested the coefficients of friction of titanium and SS brackets in conjunction with SS and ionimplanted beta-titanium arch wires. Ion-implanted beta-titanium wires generally had significantly larger coefficients of friction than SS wires that was also reflected in the severity of their wear patterns. Another study conducted by Kusy et al. ${ }^{22}$ showed no difference between the frictional values of TMA and low-friction TMA, and these values were double that of the SS wire. This study showed that ion implantation did not improve frictional properties of betatitanium wires. This might account for the high-frictional force produced by low-friction TMA with both types of brackets used in this study. Doshi et al. suggested ion implant of both the bracket slot and the arch wire demonstrated maximal reduction in frictional resistance. ${ }^{18}$ According to the results obtained in the current study, titanium brackets coupled with ion-implanted TMA wires are not recommended for sliding mechanics owing to the high-frictional resistance. In all the groups, titanium brackets showed greater mean static and kinetic frictional forces compared to SS brackets which may be attributed to the rough surface texture of the titanium brackets (Fig. 3) compared to that of SS brackets (Fig. 4) as confirmed by SEM. Similar findings were reported by Kusy et al. that titanium brackets displayed a distinct fibrous-like texture in the slot direction and were rougher compared to the SS brackets. ${ }^{14}$ In this study, titanium brackets and SS brackets produced the least static and kinetic friction with timolium wire among all the beta-titanium wires used. The SEM examination of timolium wires showed irregular patchy appearance both before and after the friction testing. Although SEM examination of timolium wires showed a rough surface texture of timolium wire, its frictional characteristics were superior to the remaining beta-titanium wires with both types of brackets. Similar findings were obtained in a study done by Kusy et al., supporting the present study. It showed that although timolium surfaces are the roughest of all beta-titanium wires, their static and kinetic coefficients of frictional values are the slickest. The stiffness and yield strength of this alpha-beta alloy are substantially greater than any of the alternative beta-titanium alloys studied. These increases coupled with a slight reduction in its flexibility might have favored a reduction in its resistance to sliding in spite of possessing a rough surface texture. ${ }^{22}$ This property makes timolium wire a suitable preference for use, with titanium brackets during retraction. In present study, the fact that timolium wire produced low friction in spite of its high surface roughness points out that frictional resistance is not proportional to the surface roughness of the wire and the same was confirmed with the SEM examinations seen in studies done by various authors. ${ }^{18,23-26}$ On the other hand, the mean static and kinetic frictional force values for SS brackets were highest with $\mathrm{Ti}-\mathrm{Nb}$ wire followed by low-friction TMA then timolium and the least was with the SS wire. This showed that SS bracket would form the best combination with SS wire owing to low friction in sliding mechanics and also lesser surface roughness. This finding was supported by Vijaylakshmi et al. who performed a similar in vitro study on metallurgical properties of SS, TMA, timolium, and $\mathrm{Ti}-\mathrm{Nb}$ arch wires with SS brackets. Although with low-friction TMA and Ti-Nb wires, greater static and kinetic friction was produced by titanium brackets compared to SS brackets, and the difference was statistically insignificant. Titanium brackets produced least friction with timolium wire followed by SS wire, and the result was statistically insignificant. This means that while using titanium brackets in nickel-sensitive patients least friction would be produced with timolium wire during retraction in sliding mechanics. Low-friction TMA and Ti-Nb would be the secondary wires of choice in such cases.

This study measured the surface friction of the materials in a dry state, while the oral cavity is a wet compartment. Therefore, the corrosive nature of salivary enzymes and inorganic salts could not be simulated. Since this was an in vitro study, the attractive or repulsive nature of the material toward plaque and calculus also could not be studied. Although the study result reported the frictional resistance to be lowest for titanium brackets with timolium wire, the availability and affordability of these alloys at all the places remained inconclusive. There needs a further study with an economic and easily available materials.

\section{ConClusion}

In all groups, titanium brackets showed greater mean static and kinetic frictional forces compared to SS brackets. For titanium brackets, the least friction was produced with timolium wire followed by SS wire then low-friction TMA and highest with $\mathrm{Ti}-\mathrm{Nb}$ wire during retraction in sliding mechanics. However, in nickel-sensitive patients where titanium brackets are used as an 
alternative, the wire of choice would be timolium wire. Low-friction TMA and $\mathrm{Ti}-\mathrm{Nb}$ would be the secondary wires of choice with titanium brackets.

\section{Clinical Significance}

The frictional force generated at the bracket-wire interface during sliding has always been a distress to the orthodontic treatment. The SS brackets and wires are proved to pose the least resistance among the available materials in orthodontics. The corrosive nature of SS and nickel sensitivity are the matter of concern toward the patients. Though esthetic, nonallergic, and do not corrode, the plastic and ceramic brackets have its own disadvantages including very high frictional resistance. The nickel-free titanium metal brackets are the material of choice in this scenario. This study evaluated the wire of choice which could generate least frictional resistance with titanium brackets. Timolium wire showed least resistance followed by TMA and Ti-Nb. This study result will be proven to be beneficial in clinical orthodontics while using sliding mechanics in nickel-sensitive patients.

\section{References}

1. Burrow SJ. Friction and resistance to sliding in orthodontics: a critical review. Am J Orthod Dentofacial Orthop 2009;135(4):442-447. DOI: 10.1016/j.ajodo.2008.09.023.

2. Kapur R, Sinha PK, Nanda RS. Comparison of frictional resistance in titanium and stainless steel brackets. Am J Orthod Dentofacial Orthop 1999;116(3):271-274. DOI: 10.1016/s0889-5406(99)70237-4.

3. Kusy RP, O'grady PW. Evaluation of titanium brackets for orthodontic treatment: part II-the active configuration. Am J Orthod Dentofacial Orthop 2000;118(6):675-684. DOI: 10.1067/mod.2000.97818.

4. Ortiz AJ, Fernández E, Vicente A, et al. Metallic ions released from stainless steel, nickel-free, and titanium orthodontic alloys: toxicity and DNA damage. Am J Orthod Dentofacial Orthop 2011;140(3): e115-e122. DOI: 10.1016/j.ajodo.2011.02.021.

5. Oh KT, Kim KM. Iron release and cytotoxicity of stainless steel wires. Eur J Orthod 2005;27(6):533-540. DOI: 10.1093/ejo/cji047.

6. Hwang CJ, Shin JS, Cha JY. Metal release from simulated fixed orthodontic appliances. Am J Orthod Dentofacial Orthop 2001;120(4):383-391. DOI: 10.1067/mod.2001.117911.

7. Matos de Souza R, Nickel MML. Chromium and iron levels in saliva of patients with simulated fixed orthodontic appliances. Angle Orthod 2008;78(2):345-350. DOI: 10.2319/111806-466.1.

8. Genelhu MCLS, Marigo M, Alves-Olivera LF, et al. Characterization of nickel-induced allergic contact stomatitis associated with fixed orthodontic appliances. Am J Orthod Dentofacial Orthop 2005;128(3):378-381. DOI: 10.1016/j.ajodo.2005.03.002.

9. Park HY, Shearer TR. In vitro release of nickel and chromium from simulated orthodontic appliances. Am J Orthod 1983;84(2):156-159. DOI: 10.1016/0002-9416(83)90180-X.
10. Sfondrini MF, Cacciafesta V, Maffia E, et al. Nickel release from new conventional stainless steel, recycled, and nickel-free orthodontic brackets: an in vitro study. Am J Orthod Dentofacial Orthop 2010;137(6):809-815. DOI: 10.1016/j.ajodo.2008.07.021.

11. Gursoy UK, Sokucu O, Uitto VJ, et al. The role of nickel accumulation and epithelial cell proliferation in orthodontic treatment-induced gingival overgrowth. Eur J Orthod 2007;29(6):555-558. DOI: 10.1093/ ejo/cjm074.

12. Drescher D, Bourauel C, Schumacher HA. Frictional forces between bracket and arch wire. Am J Orthod Dentofacial Orthop 1989;96(5):397-404. DOI: 10.1016/0889-5406(89)90324-7.

13. Sachdeva $\mathrm{CL}$, Orthodontic bracket. United States Patent. Patent number 1993:5,232,361.

14. Kusy RP, Whitley JQ, Ambrose WW, et al. Evaluation of titanium brackets for orthodontic treatment: Part I. The passive configuration. Am J Orthod Dentofacial Orthop 1998;114(5):558-572. DOI: 10.1016/ s0889-5406(98)70176-3.

15. Nanda R. Biomechanics in Clinical Orthodontics. W.B Saunders Company; 1997.

16. Proffit WR, Fields HW, Sarver DM. Contemporary Orthodontics. 4th ed., St Louis: Mosby; 2007.

17. Gioka C, Bourauel C, Zinelis S, et al. Titanium orthodontic brackets: structure, composition, hardness and ionic release. Dent Mater 2004;20(7):693-700. DOI: 10.1016/j.dental.2004.02.008.

18. Doshi UH, Bhad-Patil WA. Static frictional force and surface roughness of various bracket and wire combinations. Am J Orthod Dentofacial Orthop 2011;139(1):74-79. DOI: 10.1016/j.ajodo.2009.02.031.

19. Verstrynge A, Van Humbeeck J, Willems G. In-vitro evaluation of the material characteristics of stainless steel and beta-titanium orthodontic wires. Am J Orthod Dentofacial Orthop 2006;130(4):460470. DOI: 10.1016/j.ajodo.2004.12.030.

20. Brantley WA, Eliades T. Orthodontic Materials: Scientific and Clinical Aspects. New York: Thieme Stuttgart; 2001.

21. Michelberger DJ, Eadie RL, Faulkner MG, et al. The friction and wear patterns of orthodontic brackets and archwires in the dry state. Am J Orthod Dentofacial Orthop 2000;118(6):662-674. DOI: 10.1067/ mod.2000.105529.

22. Kusy RP, Whitley JQ, de Araújo Gurgel J. Comparisons of surface roughnesses and sliding resistances of 6 titanium-based or TMA-type archwires. Am J Orthod Dentofacial Orthop 2004;126(5):589-603. DOI: 10.1016/j.ajodo.2003.09.034.

23. Kusy RP, Whitley JQ, Mayhew MJ, et al. Surfaceroughness of orthodontic archwires via laser spectroscopy. Angle Orthod 1988; 58(1):33-45. DOI: 10.1043/0003-3219(1988)0582.0.CO;2.

24. Tidy DC. Frictional forces in fixed appliances. Am J Orthod Dentofacial Orthop 1989;96(3):249-254. DOI: 10.1016/0889-5406(89)90462-9.

25. Kusy RP, Whitley JQ. Effects of surface roughness on the coefficients of friction in model orthodontic systems. J Biomech 1990;23(9):913-925. DOI: 10.1016/0021-9290(90)90356-8.

26. Prososki RR, Bagby MD, Erickson LC. Static frictional force and surface roughness of nickel-titanium arch wires. Am J Orthod Dentofacial Orthop 1991;100(4):341-348. DOI: 10.1016/0889-5406(91)70072-5. 